\section{Successful treatment of progressive macular hypomelanosis}

\author{
Nicholas Leonard, ${ }^{1}$ Steven Krueger, ${ }^{1}$ \\ Mehdi Rashighi ${ }^{1,2}$ \\ ${ }^{1}$ University of Massachusetts Medical \\ School, Department of Dermatology; \\ ${ }^{2}$ Vitiligo Clinic and Research Center, \\ University of Massachusetts Medical \\ School, Worcester, USA
}

\begin{abstract}
Progressive Macular Hypomelanosis (PMH) is a common but often misdiagnosed disorder of acquired hypopigmentation. An adolescent female presented with irregular, hypopigmented patches ultimately diagnosed as PMH. Complete repigmentation was achieved with narrowband UVB phototherapy, benzoyl peroxide wash, and clindamycin lotion.
\end{abstract}

\section{Case Report}

An otherwise healthy 15-year-old Indian-American female presented with three years of progressive, patchy skin discoloration on the back and legs. The affected areas were asymptomatic and without scaling or redness. Prior treatment included triamcinolone $0.1 \%$ cream with no improvement. No other topical steroids or immunomodulating drugs were attempted previously. The patient's past medical history included asthma but no other atopic or allergic conditions. Physical examination showed ill-defined, irregular patches of hypopigmentation, mainly on the lower back and posterior thighs (Figure 1). No depigmented lesions or poliosis were present. Before being referred to our clinic, the patient underwent a skin biopsy on the left lateral lower back that was originally thought to be suggestive of post-inflammatory dyspigmentation. This biopsy demonstrated a normal epidermis and an upper dermis with mild vascular ectasia and a sparse mononuclear infiltrate, with occasional melanophages. A periodic acid-Schiff stain revealed no fungal elements. There were no atypical lymphocytes to suggest hypopigmented mycosis fungoides, and no granulomatous infiltrate to suggest leprosy, sarcoidosis, or fungal infection. Special stains revealed a normal number of epidermal melanocytes, helping to rule out vitiligo. The presence of basal layer pigment also helped to rule out chemical leukoderma. Based on this combination of histological and clinical data, the patient was diagnosed with Progressive Macular Hypomelanosis (PMH). Treatment included the daily application of benzoyl peroxide wash and $1 \%$ clindamycin lotion, in addition to narrowband UVB (nbUVB) three times a week. After three months, the patient showed a nearly complete resolution (Figure 2).

\section{Discussion}

$\mathrm{PMH}$ is a common skin disorder that typically presents as asymptomatic, ill-defined, non-scaly, hypopigmented macules on the trunk and proximal extremities that often become confluent towards the midline. ${ }^{1}$ Although classically absent, facial lesions have been reported in a recent case study of $\mathrm{PMH} .^{2}$ This condition is most common in young females and those with darker skin tones. ${ }^{1,3}$ Although the exact etiology is unclear, it is hypothesized that the skin commensal, Propionibacterium acnes (P. acnes), recently renamed Cutibacterium acnes, is directly involved in the pathogenesis of $\mathrm{PMH}$ potentially through the production of a currently unidentified factor that disrupts melanogenesis. ${ }^{4-6}$ Commonly associated with the pathogenesis of acne vulgaris, these bacteria reside in pilosebaceous ducts which helps to explain the typical distribution of $\mathrm{PMH}$ in skin regions with a higher density of sebaceous glands. ${ }^{6}$ Quantities of P. acnes have been shown to be elevated in lesional skin of patients with $\mathrm{PMH}$ through both culture and quantitative real-time PCR. ${ }^{5} \mathrm{PMH}$ diagnosis continues to elude practitioners, and studies have yet to suggest a standard approach. A recent case study suggested that the use of Wood's lamp examination can aid diagnosis by revealing a specific follicular pattern of fluorescence, proportional to the concentration of $P$. acnes, that is not present in hypopigmented lesions caused by other etiologies. ${ }^{7}$ It is essential to differentiate this condition from other acquired hypopigmentation disorders such as sarcoidosis, leprosy, tinea versicolor, syphilis, hypopigmented mycosis fungoides, pityriasis alba, hypopigmented plaque parapsoriasis, and nutritional deficiencies in order to avoid a delay in treatment.

The literature on the ideal treatment of $\mathrm{PMH}$ has been nonconclusive to date. Initial treatment recommendations were based on a 14-week long trial that found a higher efficacy of benzoyl peroxide 5\% hydrogel at night, clindamycin $1 \%$ lotion in the morning, and UVA phototherapy for 20 minutes
Correspondence: Mehdi Rashighi, Assistant Professor of Dermatology, Director, Connective Tissue Disease Clinic and Research Center; University of Massachusetts Medical School, Department of Dermatology; Vitiligo Clinic and Research Center, Worcester, 01545 MA, USA

E-mail: mehdi.rashighi@umassmed.edu

Tel.: +1 774-455-4759

Fax: 508-856-7883

Key words: Phototherapy, pigmentary disorders, topical therapy

Acknowledgements: The authors of this case report thank the staff at UMass Medical School who participated in this patient's care.

Conflict of interest: the authors declare no potential conflict of interests.

Received for publication: 19 February 2020. Accepted for publication: 14 July 2020.

This work is licensed under a Creative Commons Attribution-NonCommercial 4.0 International License (CC BY-NC 4.0).

${ }^{\circ}$ Copyright: the Author(s), 2020

Licensee PAGEPress, Italy

Dermatology Reports 2020; 12:8509

doi:10.4081/dr.2020.8509

three times weekly compared with fluticasone $0.05 \%$ cream at night and UVA phototherapy for 20 minutes three times weekly. ${ }^{8}$ The use of nbUVB once or twice weekly for $\mathrm{PMH}$ was introduced in a study of 16 patients, where $56.2 \%$ of individuals showed more than $90 \%$ repigmentation, but with a $31 \%$ relapse rate. ${ }^{9}$ A retrospective analysis of 108 patients with PMH compared treatment with topical antimicrobials (clindamycin or benzoyl peroxide) to nbUVB twice weekly. ${ }^{10}$ In that study, $38 \%$ of patients treated with antimicrobials achieved resolution at an average of 8.5 months with no relapse, whereas $90 \%$ of patients treated with nbUVB achieved resolution at an average of only 2.5 months but had a $6 \%$ relapse rate. The authors hypothesized that nbUVB mainly serves to promote repigmentation but is less effective at longterm elimination of $C$. acnes. Therefore, a combination approach with topical antibiotics (daily application of benzoyl peroxide wash and $1 \%$ clindamycin lotion) and nbUVB phototherapy (three times weekly) was recommended. The only study comparing nbUVB alone to nbUVB with antimicrobials reported a lack of statistical power to suggest an efficacy difference. ${ }^{11}$

In conclusion, our case of PMH treated successfully with phototherapy and topical antibiotics highlights how the absence of 

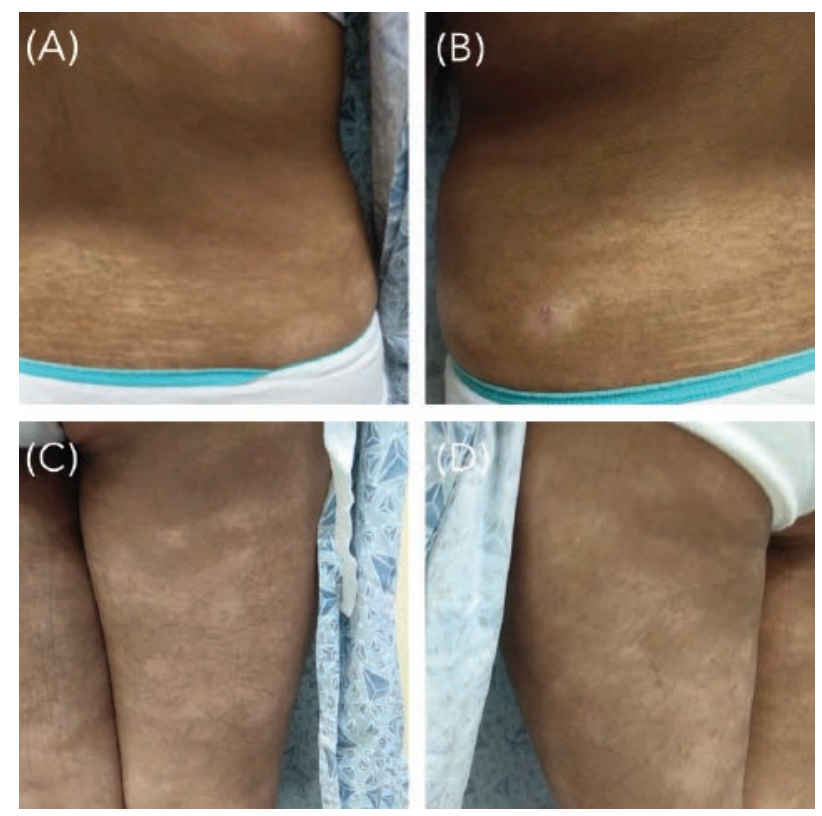

Figure 1. Pre-treatment clinical photographs showing ill-defined, irregular patches of hypopigmentation on the lower back $(\mathrm{A}, \mathrm{B})$, and back of legs $(\mathrm{C}, \mathrm{D})$.
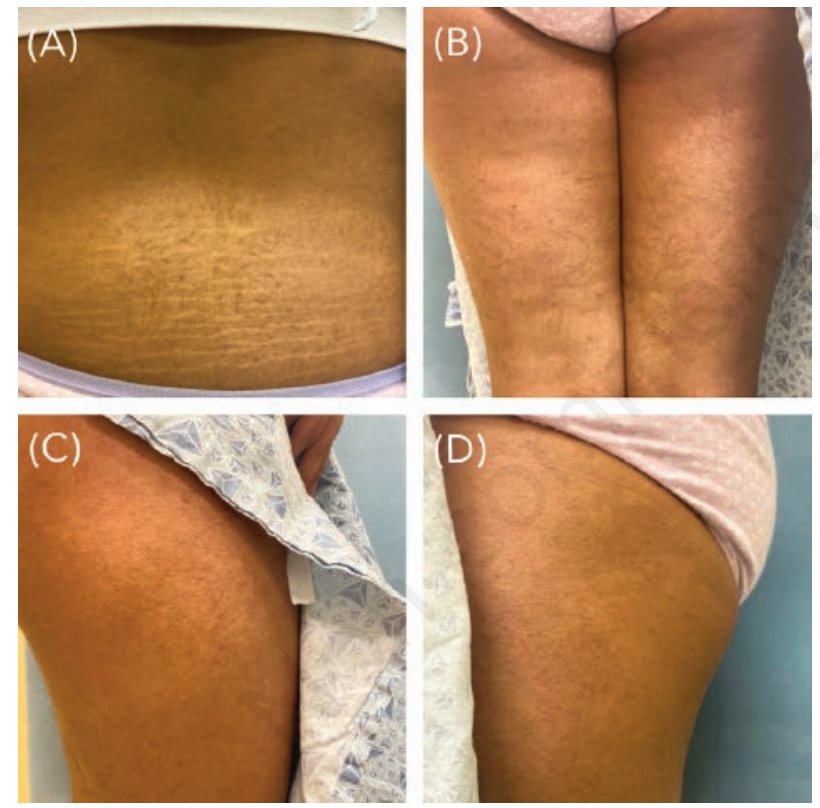

Figure 2. Post-treatment clinical photographs showing resolution of hypopigmented patches on the lower back (A), and the legs (B, C, D).

specific histological features can help to narrow down this elusive diagnosis. Furthermore, we report excellent results from a combination therapy that is hypothesized to be efficacious but has been poorly studied to date.

\section{References}

1. Relyveld GN, Menke HE, Westerhof W. Progressive macular hypomelanosis: an overview. Am J Clin Dermatol 2007;8:13-9. doi: 10.2165/00128071200708010-00002

2. Wang K, Nassef Y, Sahu J, et al. Facial involvement in progressive macular hypomelanosis. Cutis 2018;101:297300. Available from: https://mdedgefiles- live.s3.us-east-2.amazonaws.com/ files/s3 fs-public/Document/April2018/CT101004297.PDF

3. Shen LY, Kenner-Bell BM, Ricketts J, Kundu RV. Ethnic skin: kids are not just little people. Clin Dermatol 2016;34: 690-7. doi: 10.1016/j.clindermatol.2016.07.003.

4. Petersen RLW, Scholz CFP, Jensen A, et al. Propionibacterium acnes phylogenetic type III is associated with progressive macular hypomelanosis. Eur J Microbiol Immunol 2017;7:37-45. doi: $10.1556 / 1886.2016 .00040$

5. de Morais Cavalcanti SM, de França ER, Magalhães M, et al. A quantitative analysis of Propionibacterium acnes in lesional and non-lesional skin of patients with progressive macular hypomelanosis by real-time polymerase chain reaction. Braz $\mathrm{J}$ Microbiol 2011;42:423-429. doi: 10.1590/S151783822011000200002

6. Platsidaki E, Dessinioti C. Recent advances in understanding Propionibacterium acnes (Cutibacterium acnes) in acne. F1000 Res 2018;7:1953. doi: 10.12688/f1000research.15659.1

7. Pflederer RT, Wuennenberg JP, Foote C, et al. Use of woods lamp to diagnose progressive macular hypomelanosis. J Am Acad Dermatol 2017;77:e99-e100. doi: 10.1016/j.jaad.2017.05.004.

8. Relyveld GN, Kingswijk MM, Reitsma $\mathrm{JB}$, et al. Benzoyl peroxide/clindamycin/uva is more effective than fluticasone/uva in progressive macular hypomelanosis: a randomized study. J Am Acad Dermatol 2006;55:836-43. doi: 10.1016/j.jaad.2006.03.020.

9. Kim M-B, Kim G-W, Cho H-H, et al. Narrowband UVB treatment of progressive macular hypomelanosis. J Am Acad Dermatol 2012;66:598-605. doi: 10.1016/j.jaad.2011.04.01010.

Thng SG, Long VH, Chuah S, Tan VD. Efficacy and relapse rates of different treatment modalities for progressive macular hypomelanosis. Indian J Dermatol Venereol Leprol 2016;82: 673-6. doi: 10.4103/0378-6323.182797.

11. Sim J, Lee D, Lee J, Kim Y. Comparison of the clinical efficacy of nbuvb and nbuvb with benzoyl peroxide/clindamycin in progressive macular hypomelanosis. J Eur Acad Dermatol Venereol 2011;25:1318-1323. doi:10. 1111/j.1468-3083.2011.03980.x 